\title{
Effect of Current Polarity on BSCCOIAg Ceramics Textured by Electrically Assisted Laser Floating Zone
}

\author{
F.M. Costa ${ }^{1}$, S. Rasekh², N.M. Ferreira ${ }^{1}$, A. Sotelo ${ }^{2}$, J.C. Diez ${ }^{2}$, M.A. Madre ${ }^{2}$ \\ ${ }^{1}$ i3N, Dep. of Physics, University of Aveiro, Campus de Santiago, 3810-193 Aveiro, \\ Portugal \\ ${ }^{2}$ ICMA, CSIC-Universidad de Zaragoza, Maria de Luna, 3, 50018 Zaragoza, Spain
}

\begin{abstract}
Bi-2212/Ag superconducting ceramics were grown using the electrically assisted laser floating zone (EALFZ) technique. The main goal was improving the superconducting properties by applying an electrical current through the sample during the solidification process. The effect of a $100 \mathrm{~mA}$ current intensities in two configurations were studied: (i) direct current (connecting the seed rod to the positive pole and the feed to the negative one) and (ii) reverse current (positive pole connected to the feed rod and negative to the seed one). The resulting textured cylindrical bars were annealed in order to obtain the superconducting phases. A strong correlation between current polarity, grain alignment and superconducting properties was observed. The rods grown under direct current exhibit higher critical current density $\left(\sim 3000 \mathrm{~A} / \mathrm{cm}^{2}\right)$ than the ones grown under conventional method $\left(\sim 2300 \mathrm{~A} / \mathrm{cm}^{2}\right)$ or under reverse current condition $(\sim 35$ $\mathrm{A} / \mathrm{cm}^{2}$ ). The superior value obtained for the direct current results from a better grain alignment and fewer amount of second phases.
\end{abstract}

Keywords: Bi-2212; Superconductivity; Critical current; Electrical current; Laser floating zone

Corresponding author: F.M. Costa. i3N, Dep. of Physics, University of Aveiro, Campus de Santiago, 3810-193 Aveiro, Portugal. e-mail: flor@ua.pt 


\section{Introduction}

The major current limiting factors for high power application of BSCCO materials are the intrinsic granularity and the strong structural anisotropy which may be several orders of magnitude lower along the c-axis direction than in the $a b$ plane. The electrical current transport problems can be controlled to some extent by texturing the material and minimizing the amount of secondary phases [1-3]. There are several processing techniques that can be used to induce texture in the superconducting material. Among them, the laser floating zone (LFZ) technique which, due to the very localized heating, generates a very high thermal gradient at the solidification interface that promotes the grain alignment during the growth process [4]. However, due to the thermal gradient dependence on fiber diameters $[5,6]$ the maximum critical current that can be transported by fibers tends to decrease with the increase of fiber diameter due to grain alignment decreasing. The solution could be to compensate this dependence increasing the thermal gradient by the application of a dc electrical current during the solidification of the BSCCO rods $[7,8]$. This new Electrically Assisted Laser Floating Zone (EALFZ) technique proved to significantly change the solid/liquid solidification interface and, as a consequence, the resulting phase nature, crystals shape and orientation [7]. Another contribution to obtain bulk materials foreseeing high power applications is the $\mathrm{Ag}$ addition to the initial powder. In fact, previous results have demonstrated that $\mathrm{Ag}$ inclusions improve the microstructure, reducing the porosity and increasing the mechanical properties along with an improvement of electrical and magnetic properties $[9,10]$.

In the present work, the EALFZ technique will be applied to the Bi-2212/Ag material in order to study the effect of electrical current polarity on microstructural characteristics, phase development, and transport properties.

\section{Experimental Procedure}

Cylindrical precursors with $\mathrm{Bi}_{2.02} \mathrm{Sr}_{2.02} \mathrm{Ca}_{0.98} \mathrm{Cu}_{1.99} \mathrm{O}_{x} / 2.9$ wt. \% Ag nominal composition (Nexans SuperConductors $\mathrm{GmbH}$ ) were obtained by cold isostatic pressing at $200 \mathrm{MPa}$ during 1 minute to be used as feed and seed rods for the 
EALFZ process. $A \mathrm{CO}_{2}$ laser $(\lambda=10.6 \mu \mathrm{m} ; 200 \mathrm{~W})$ was used to grow rods with about $100 \mathrm{~mm}$ in length and $2.5 \mathrm{~mm}$ in diameter at $30 \mathrm{~mm} / \mathrm{h}$, using a relative rotation of $18 \mathrm{rpm}$ between seed and feed rods. Three distinct growth conditions were accomplished: (i) no current application through the solidification interface (conventional LFZ process); (ii) a dc electric current of $100 \mathrm{~mA}$ (direct current) with the positive pole connected to the seed rod; (iii) a reverse dc electric current of 100 $\mathrm{mA}$ connecting the negative pole to the seed rod. All rods were subjected to a two steps heat treatment: $860{ }^{\circ} \mathrm{C}(60 \mathrm{~h})$ and $800{ }^{\circ} \mathrm{C}(12 \mathrm{~h})$. The rod microstructure analysis and phase identification were performed by scanning electron microscopy (SEM), provided with energy dispersive X-ray (EDX) spectroscopy. The X-ray diffraction (XRD) analysis was used as a complementary technique for phase identification. Pole figures to the (110) plane were obtained to quantify the grain alignment degree.

The four-point probe was applied to measure the resistivity as a function of temperature and the critical current at $77 \mathrm{~K}$ in a bath of liquid nitrogen, using the critical electric field criterion of $1 \mu \mathrm{V} / \mathrm{cm}$.

\section{Results and Discussion}

After annealing, all growth conditions lead to polycrystalline and polyphasic rods, as is shown in Fig. 1, where representative microstructures of the annealed rods grown with no current $(\operatorname{rod} A)$, reverse current $(\operatorname{rod} B)$ and direct current $(\operatorname{rod} C)$, are depicted. A strong dependence of current polarity can be observed in what concerned to the grain alignment, secondary phase nature and amount. The rods grown under a conventional LFZ process, i.e., without electrical current $(\operatorname{rod} A)$, are mainly composed by platelet-like 2212 crystals, together with Ag particles and 1/1 cuprate (Fig. 1(a), (b)). The secondary phases are mainly localized at the rod periphery due to the faster cooling of this region that promotes the development of the most refractory phases [11]. Under the reverse electrical current condition (rod B) the amount of secondary phases (1/1, 2/1 and a copper free phase) significantly increases and simultaneously the preferential grain alignment disappear leading to an almost randomly oriented microstructure (Fig. 1(c), (d)). Contrarily, the rod 
grown with direct current (rod C) shows the same type of phases observed in the $\operatorname{rod} A$, grown without current, but with smaller amount of $1 / 1$ phase. Moreover, the superconducting grains in rod $\mathrm{C}$ exhibit higher alignment (Fig. 1(e), (f)) than in rod A due to the current application.

The effect of current application on rod texture is well visible on pole plots represented in Fig. 2. Taking into account that the Bi-2212 crystals grow preferentially with the ab plane parallel to the rod axis, the (110) plane at $23.1^{\circ}$ was selected to measure the rods texture [12]. This way, the pole plots in X-ray diffraction were acquired from polished transversal rod sections. The number of (110) reflections is significantly superior in rod C (Fig. 2(b)), which corresponds to the sample grown by EALFZ with direct current.

The temperature dependence of the electrical resistivity for all rods shows the same transition temperature from the normal to the superconducting state, Fig. 3. Despite the critical temperature being independent of growth conditions, the same is not happening with the electrical resistivity at room temperature. In fact, the value for the rod grown under reverse current $(\operatorname{rod} B)$ is much higher than for the other rods. However, the biggest difference between the three samples type is revealed by the critical current values obtained from the electric field as a function of current density data for the annealed rods. These values, determined at $77 \mathrm{~K}$, are shown in Table 1. The difference of the critical current density values is justified by the preferential grain orientation of superconducting crystals in rods $A$ and $C$ and by the random orientation of grains in the rod B. Also, the different amount of secondary phases contributes to this difference.

These results demonstrate the important influence of the electrical current application during the crystallization process of superconducting materials by laser floating zone process. In fact, the solidification under an electrical field changes the solute segregation at the solid-liquid interface due to the competition of constitutional undercooling associated with the steep thermal gradient characteristic of the LFZ process, and the electromigration effect due to the selective ionic drift under the electrical current [7]. As a consequence of both phenomena, an intensification of grain alignment could be obtained, together with 
the favorable development of superconducting phases if the correct polarity is used during the EALFZ growth. In opposition, the preferential grain alignment could disappear and the number of non-superconducting phases increases if a reverse current is selected.

\section{Conclusions}

The application of an electrical current during processing of $\mathrm{Bi}-2212 / \mathrm{Ag}$ using the laser floating zone can be used to promote changes in the amount of secondary phases and grain orientation. An intense dependence of current polarity was observed due to modifications on the ionic mobility and on the temperature gradient at the solidification interface. When the positive pole of electrical current is connected to the feed rod, the number of non-superconducting phases increases and the preferential grain alignment disappear. In opposition, when the polarity is changed (direct current) an intensification of grain alignment is observed together with an increase of superconducting phases amount, which leads to an increase of critical current density of about $30 \%$, compared with samples grown with no applied current.

\section{Acknowledgements}

The authors are thankful to FCT for the project PEst-C/CTM/LA0025/2011. The Spanish MICINN-FEDER (Projects MAT2008-00429 and AIB2010-PT-00247), MINECO-FEDER (Project MAT2011-22719), the Universidad de Zaragoza (UZ2011-TEC-03), and the Gobierno of Aragón (research consolidate groups T12 and T87) are acknowledged for financial support. Sh. Rasekh acknowledges a JAE-PreDoc2010 grant from the MICINN-CSIC. 


\section{References}

1. Feigelson, R.S., Gazit, D., Fork D, K., Geballe, T.H.: Science 240, 1642 (1988)

2. Costa, F.M., Silva, R.F., Vieira, J.M.: Physica C 289, 171-176 (1997)

3. Angurel, L.A., Díez, J.C., Fuente, G.F., Gimeno, F., Lera, F., López-Gascón, C., Martínez, E., Mora, M., Navarro, R., Sotelo, A., Andrés, N., Recuero, S., Arroyo, M.P.: Phys. Status Solidi A 203, 2931-2937 (2006)

4. Andreeta, M.R.B., Andreeta, E.R.M., Hernandes, A.C., Feigelson, R.S.: J. Cryst. Growth 234, 759-761 (2002)

5. Brice, J.C.: J. Cryst. Growth 42, 427-430 (1977)

6. Vieira, J.M., Silva, R.A., Silva, R.F., Costa, F.M.: Appl. Surf. Sci. (2012). doi:10.1016/j.apsusc.2011.11.054

7. Carrasco, M.F., Silva, R.F., Vieira, J.M., Costa, F.M.: Supercond. Sci. Technol. 17, 612-619 (2004)

8. Carrasco, M.F., Silva, R.A., Silva, R.F., Amaral, V.S., Costa, F.M.: Physica C 460-462, 1347-1348 (2007)

9. Sotelo, A., Madre, M.A., Diez, J.C., Rasekh, Sh., Angurel, L.A., Martínez, E.:

Supercond. Sci. Technol. 22, 034012 (2009)

10. Sotelo, A., Mora, M., Madre, M.A., Diez, J.C., Angurel, L.A., Fuente, G.F.: J.

Eur. Ceram. Soc. 25, 2947 (2005)

11. Carrasco, M.F., Silva, R.F., Vieira, J.M., Costa, F.M.: Supercond. Sci. Technol.

22, 065016 (2009)

12. Costa, F.M., Silva, R.F., Vieira, J.M.: Physica C 289, 161-170 (1997) 
Table 1. Values of applied electrical current $(I)$ during the EALFZ growth and the critical current $\left(I_{c}\right)$, and critical current density $\left(J_{c}\right)$ obtained for the three growth conditions and the corresponding rod diameter $(\varnothing)$

\begin{tabular}{|c|c|c|c|}
\hline$I(\mathrm{~mA})$ & $I_{c}(\mathrm{~A})$ & $\phi(\mathrm{mm})$ & $J_{c}\left(\mathrm{~A} / \mathrm{cm}^{2}\right)$ \\
\hline 0 & 117 & 2.55 & 2294 \\
\hline-100 & 1.5 & 2.37 & 34 \\
\hline+100 & 135 & 2.40 & 3004 \\
\hline
\end{tabular}




\section{Figure captions}

Figure 1. Scanning electron microscopy images of polished longitudinal sections of Bi-2212 annealed rods grown without electrical current, rod A (a) and (b), under reverse current of $-100 \mathrm{~mA}$, rod $B(\mathbf{c})$ and (d) and under direct current of $+100 \mathrm{~mA}$, $\operatorname{rod} \mathrm{C}(\mathbf{e})$ and $(\mathbf{f})$.

Figure 2. Pole figures of Bi-2212 phase (110) plane performed on (a) rod A grown by conventional laser floating zone process (no current application), and (b) rod C with direct current $(+100 \mathrm{~mA})$.

Figure 3. Electrical resistivity as a function of temperature of heat-treated rods grown under different conditions. 
Figure 1

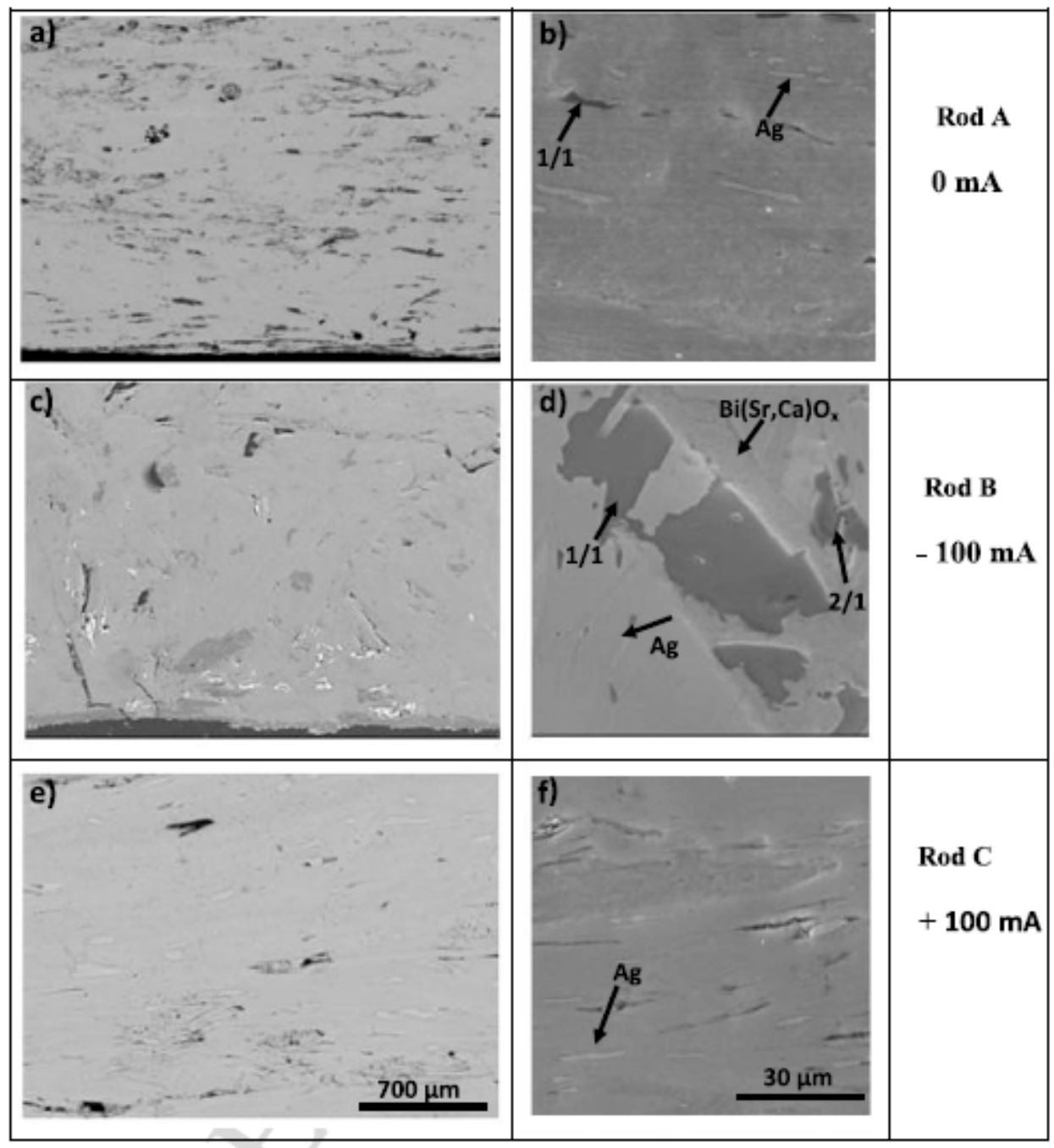


Figure 2
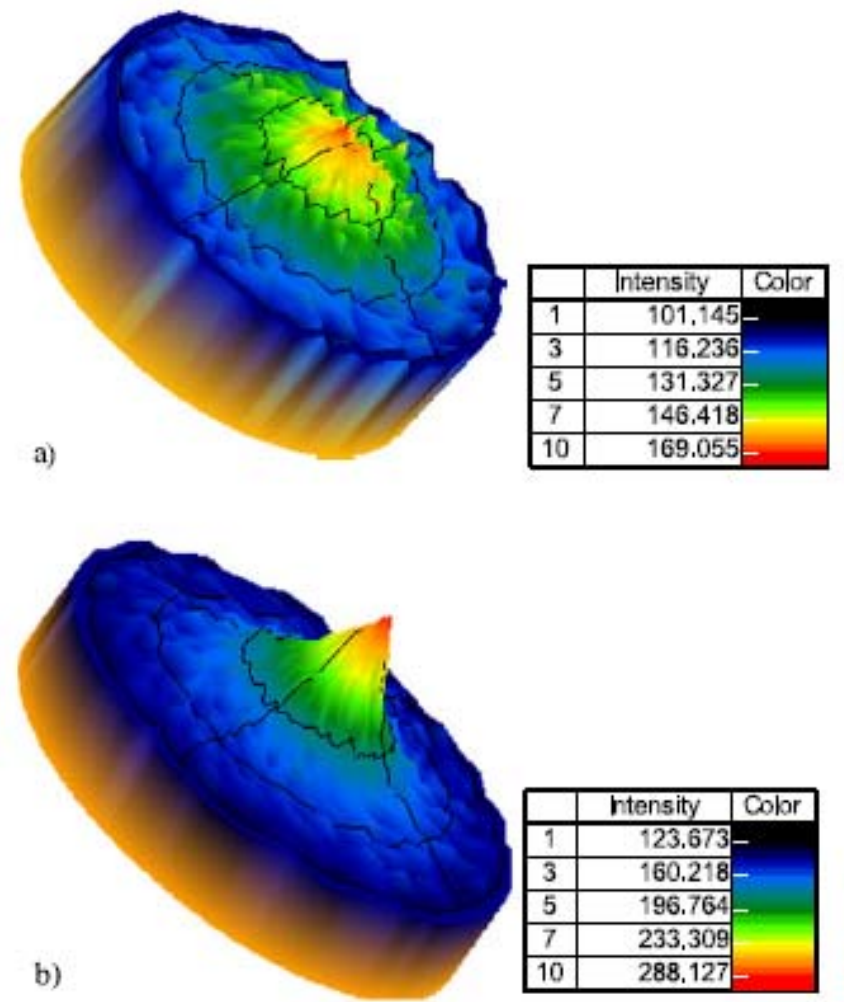
Figure 3

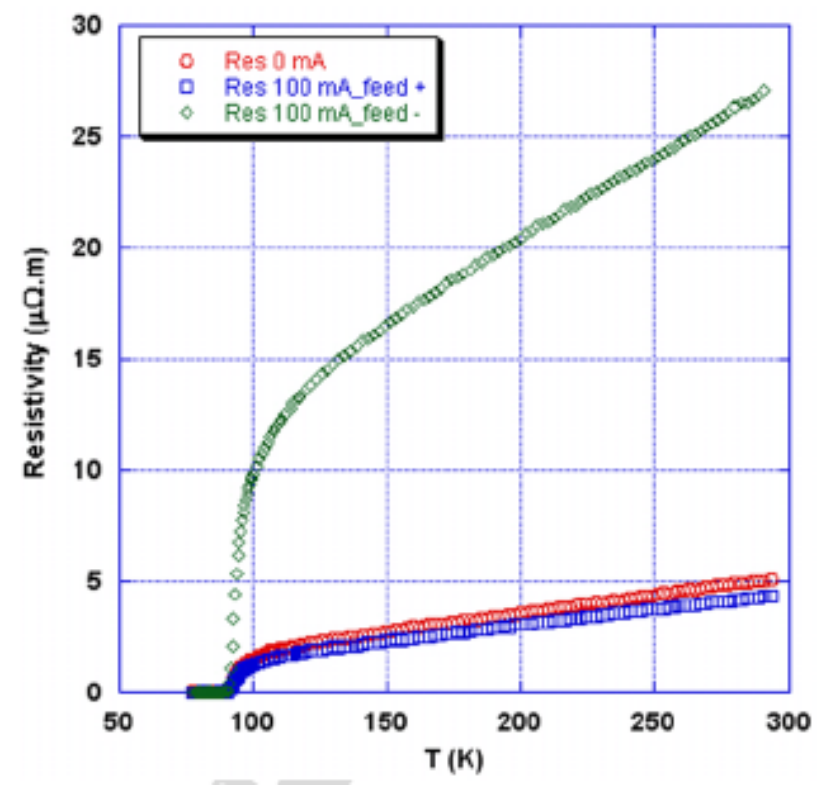

